\title{
Cyclotron spatial autoresonance acceleration model
}

\author{
Valeriy D. Dugar-Zhabon and Eduardo A. Orozco \\ Universidad Industrial de Santander, A.A. 678 Bucaramanga, Colombia
}

(Received 15 July 2008; published 2 April 2009)

\begin{abstract}
An autoresonance electron acceleration phenomenon in the combined steady-state inhomogeneous magnetic and microwave fields is analytically studied. Equations describing the evolution of the phase shift between the particle velocity and the microwave electric field, total energy, and longitudinal velocity of the electron are obtained. Linear and parabolic profiles of the magnetic field are examined. It is shown that the proper choice of the magnetic heterogeneity degree, the microwave electric field value, and initial electron velocity can retain the electron in the acceleration phase band. The results obtained in this work show a complete agreement with our previous autoresonance results obtained through simulations of the relativistic Newton-Lorentz equation.
\end{abstract}

DOI: 10.1103/PhysRevSTAB.12.041301

PACS numbers: 29.20.D-, 29.27.-a

\section{INTRODUCTION}

It is well known that the cyclotron autoresonance or selfsustenance regime for an electron is performed by continuous maintaining of equality between the frequency of the electromagnetic field and the cyclotron frequency. The autoresonance electron-microwaves interaction in homogeneous magnetic fields was realized by increasing in time of the magnetic field level at a rate which permits to keep the cyclotron frequency value equal to the microwaves frequency despite the relativistic electron mass rise [1-8]. A cyclotron resonance acceleration of the electrons in a magnetostatic guide field by a traveling electromagnetic wave with adiabatically varying parameters was named spatial autoresonance cyclotron accelerator $[9,10]$. The electron acceleration to high energies by microwaves was also found in the numerical experiments on plasma dynamics in an electron cyclotron resonance (ECR) minimum-B trap [11], which was attributed to a selfsustenance of the ECR conditions on some part of the electron trajectory. Plasma heating by pulse microwaves in an adiabatic mirror magnetic trap observed in the experiments [12] was attributed to a cyclotron autoresonance interaction of electrons with microwaves. We have recently reported on the possibility of self-sustenance of the ECR conditions in the stationary inhomogeneous magnetic fields at a fixed microwave field frequency through a numerical solution of the relativistic Newton-Lorentz equation in the one-particle approximation [13]. This type of cyclotron resonance speeding up of electrons can be named as spatial autoresonance acceleration (or SARA).

In the present paper, we analyze analytically the SARA phenomenon in a single particle approximation. The basic set of the SARA equations which describe the evolution of the phase, energy, and longitudinal velocity of the electron are developed. The SARA electron-microwave interaction significantly differs from the other types of autosustenance acceleration: it occurs in the inhomogeneous magnetic field. The fact that the diamagnetic force is presented in the SARA concept but not found in the GYRAC concept $[5,6]$ is a most remarkable difference between these acceleration mechanisms. For the SARA concept, the diamagnetic force is one of the important factors limiting the energy which can be achieved. The analytical results were verified through numerical experiments under the conditions which were used in our previous SARA simulations [13].

\section{PHYSICAL SCHEME AND THEORETICAL MODEL}

A physical scheme for the SARA phenomenon realization is shown in Fig. 1. A cavity of cylindrical geometry (1) is placed inside the current coil set (2) which forms a static magnetic field of the desired profile. In the cavity, a $\mathrm{TE}_{11 p}$ mode (with $p=1,2,3 \ldots$ ) can be excited (3). An electron

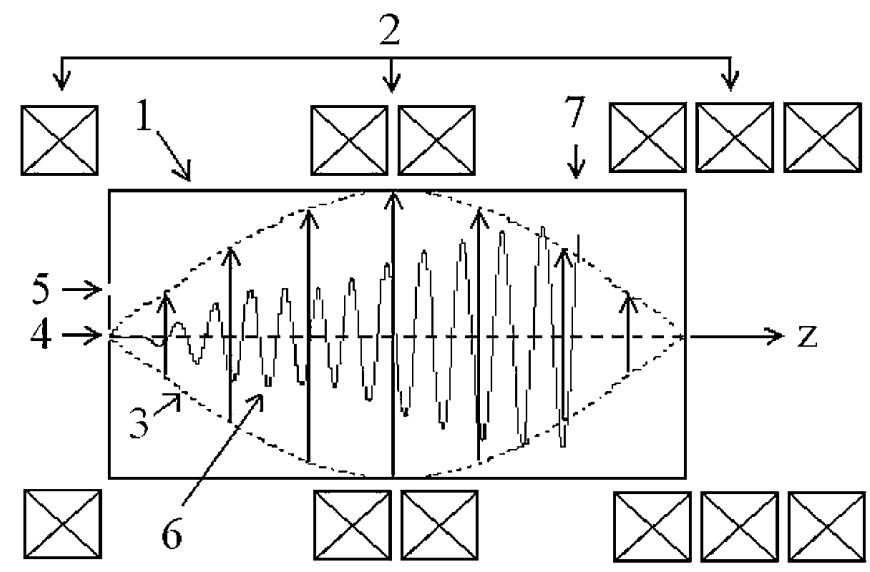

FIG. 1. A physical model scheme: 1-cavity, 2-magnetic coils, 3-electric field profile (particular case of $\mathrm{TE}_{111}$ mode), 4-on-axis injection point, 5-off-axis injection point, 6-electron trajectory, 7-stop plane. 
with the initial energy of $W_{0}$ can be injected both along the cavity axis (4) and through an off-axis orifice (5). The cavity axis which coincides with the magnetic field axis is taken as the $z$ axis.

The evolution of the magnetic field along the $z$ axis is expressed as

$$
\vec{B}(0, z)=\vec{B}_{0}\left[\gamma_{0}+b(z)\right]
$$

where $B_{0}=m_{0} \omega / e$ is the magnetic induction corresponding to the exact cyclotron resonance for the electron restmass $m_{0}, \omega$ is the electromagnetic field frequency, $\gamma_{0}=$ $\left[1-\left(v_{z 0} / c\right)^{2}\right]^{-1 / 2}$ is the initial relativistic factor, $v_{z 0}$ and $c$ are the initial electron and light velocities, respectively. The coordinate origin $z=0$ is located on the left-hand lateral side of the cavity (see Fig. 1). The longitudinal magnetic component profile is determined by the dimensionless function $b(z)$, assuming $b(0)=0$. The spatial magnetic configuration $\vec{B}(r, z)$ is deduced from the equation $\bar{\nabla} \cdot \vec{B}=0$ under the condition inside the cavity $\partial B_{z} / \partial r \cong 0$ :

$$
\vec{B}(r, z)=-(1 / 2)\left[r d B_{z}(z) / d z\right] \hat{r}+B_{z}(z) \hat{z},
$$

where

$$
B_{z}(r, z) \cong B_{z}(z)=B_{0}\left[\gamma_{0}+b(z)\right] .
$$

The $\mathrm{TE}_{11 p}$ cylindrical mode excited in the cavity is given by the expressions

$$
\begin{gathered}
E_{r}=2 E_{0}(1 / h r) J_{1}(h r) \sin \theta \sin (p \pi z / d) \cos \omega t, \\
E_{\theta}=2 E_{0} J_{1}^{\prime}(h r) \cos \theta \sin (p \pi z / d) \cos \omega t,
\end{gathered}
$$

where $h=S_{11} / a, S_{11}=1.841$, and $a$ is the radius of the cavity; $E_{0}$ is the microwave amplitude and $d$ is the cavity length. When $h r<1$, the Bessel function is approximated as $J_{1}(h r) \cong h r / 2$. Then Eqs. (4) and (5) are reduced, respectively, to

$$
\begin{aligned}
& E_{r}=E_{0} \sin \theta \sin (p \pi z / d) \cos \omega t, \\
& E_{\theta}=E_{0} \cos \theta \sin (p \pi z / d) \cos \omega t .
\end{aligned}
$$

One can see that the $\mathrm{TE}_{11 p}$ mode is well approximated by a stationary plane wave. An electron moving along the $z$ axis interacts effectively with the right-hand polarized TE standing wave if the phase shift between the electric field and the transversal velocity is maintained between $\pi / 2$ and $3 \pi / 2$. This phase shift denoted as $\varphi$ (see Fig. 2) is introduced in the expression for the electric field:

$$
\vec{E}=E_{0}(\sin \varphi \hat{r}+\cos \varphi \hat{\theta})|\sin (p \pi z / d)| .
$$

In expression (8), the third factor is given in the absolute form because the microwave electric field direction has been taken into account in the phase shift $\varphi$. The coordinate system for the off-axis injection case is shown in Fig. 3.

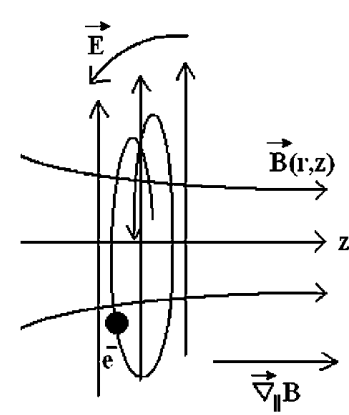

(a)

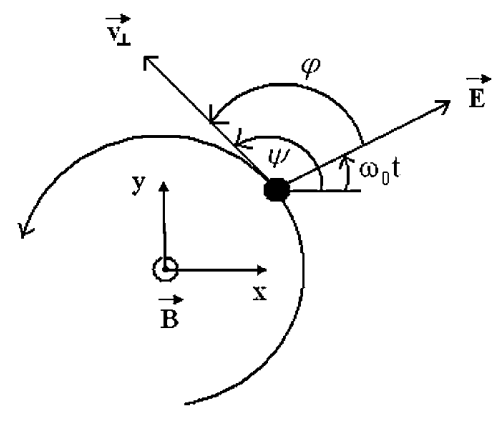

(b)
FIG. 2. (a) Mutual dispositions of the particle trajectory, electric, and magnetic fields; (b) electron trajectory projection onto the transverse plane and definitions of the angles which characterize the electron motion: $\omega_{c}=d \psi / d t$, where $\psi$ is the absolute phase of the transversal velocity $\vec{v}_{\perp}, \varphi$ is the angle between $\vec{v}_{\perp}$ and $\vec{E}$.

In the $S^{\prime}\left(x^{\prime}, y^{\prime}\right)$ coordinate system, where the point $0^{\prime}$ is the guide center of the electron injected at a point $r_{0} \neq 0$, the magnetic field components are

$$
\begin{aligned}
B_{r}^{\prime} & =-(1 / 2)\left(r^{\prime}+r_{0} \cos \theta^{\prime}\right)\left(d B_{z}^{\prime} / d z\right), \\
B_{\theta}^{\prime} & =(1 / 2)\left(r_{0} \sin \theta^{\prime}\right)\left(d B_{z}^{\prime} / d z\right), \\
B_{z}^{\prime} & =B_{z} .
\end{aligned}
$$

For the off-axis injection case, the electric field can be expressed in a form analogous to expression (8) if the inequality $r^{\prime}+r_{0}<a / S_{11}$, which is the case in the present work, is satisfied. The motion equation of an electron with the momentum $\vec{p}$ and the velocity $\vec{v}(v=\beta c$ and $p=$ $\beta \gamma m_{0} c$ ) in the combined field composed of the inhomogeneous magnetic field $\vec{B}^{\prime}(r, z)$ and the microwave electric

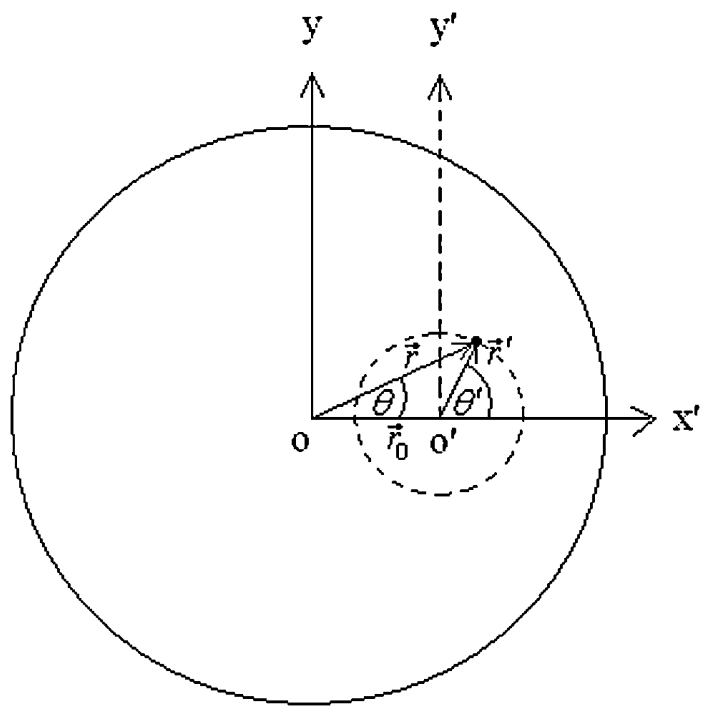

FIG. 3. Coordinate scheme for the off-axis electron injection case. 
field $\vec{E}^{\prime}$ is

$$
d \vec{p}^{\prime} / d t=\vec{F}^{\prime}=-e \vec{E}^{\prime}-e \overrightarrow{\boldsymbol{v}}^{\prime} \times \vec{B}^{\prime}+\vec{F}_{S},
$$

where $\vec{F}_{S}$ is the synchrotron radiation force which is disregarded here because the electron energies for the chosen simulations are not expected to be high enough.

Substituting Eqs. (8) and (9) in Eq. (10) and taking into account that $B_{r}^{\prime} / B_{z}^{\prime} \ll 1$ and $v_{r}^{\prime} / v_{\theta}^{\prime} \ll 1$, we obtain $v_{z}^{\prime} B_{\theta}^{\prime} / v_{\theta}^{\prime} B_{z}^{\prime} \ll 1$. Therefore, the force components can be written as

$$
\begin{gathered}
F_{r}=-e v_{\perp}^{\prime} B_{z}^{\prime}-e E_{0}\left|\sin \left(p \pi z^{\prime} / d\right)\right| \sin \varphi^{\prime}, \\
F_{\theta}=-e E_{0}\left|\sin \left(p \pi z^{\prime} / d\right)\right| \cos \varphi^{\prime}, \\
F_{z}=\left\{-\frac{m_{0} v_{\perp}^{\prime 2}}{2 B_{0}\left[\omega_{c}^{\prime}(z) / \omega\right]}-\left(\frac{e v_{\perp} r_{0}}{2}\right) \sin \left(\varphi^{\prime}+\omega t\right)\right\}\left(\frac{d B_{z}^{\prime}}{d z}\right),
\end{gathered}
$$

where $\omega_{c}(z)=v_{\perp}^{\prime}(z) / r^{\prime}(z)\left(v_{\perp}^{\prime} \cong v_{\theta}^{\prime}\right.$ and $\left.r^{\prime} \neq 0\right)$ is the local electron cyclotron frequency. Equation (11) is defined as the centripetal force, Eq. (12) is related to the energy variation, and Eq. (13) is the diamagnetic force which acts in the direction opposite to the magnetic field gradient and impedes the advance of the electrons into a higher magnetic field. In the particular case of the exact resonance $\omega_{c}^{\prime}(z)=\omega$, Eq. (13) is reduced to the equation which corresponds to the case when the electric field is absent. One can see that the spatial self-sustained acceleration is different from the other autoresonance types because in the inhomogeneous magnetic field there appears a diamagnetic force which is a function of the particle position. To simplify the presentation of the expressions here and further on, the upper index, which marks the $S^{\prime}\left(x^{\prime}, y^{\prime}\right)$ coordinate system values, is omitted.

From Eq. (11) we get

$$
\gamma m_{0} v_{\perp}^{2} / r=e v_{\perp} B_{z}(z)+e E_{0}|\sin (p \pi z / d)| \sin \varphi,
$$

where

$$
\gamma=\left[1-\left(v_{\perp}^{2}+v_{z}^{2}\right) / c^{2}\right]^{-1 / 2} .
$$

Using Eqs. (3), (14), and (15) and the dimensionless electric field $g_{0}=E_{0} / B_{0} c$, the local electron cyclotron frequency $\omega_{c}(z)$ can be written as

$$
\begin{aligned}
\omega_{c}(z) / \omega= & \gamma^{-1}\left[\gamma_{0}+b(z)\right] \\
& +g_{0} \gamma^{-1}\left[\left(1-\gamma^{-2}\right)-\left(v_{z} / c\right)^{2}\right]^{-1 / 2} \\
& \times|\sin (p \pi z / d)| \sin \varphi .
\end{aligned}
$$

The phase-shift evolution is described by the expression

$$
\dot{\varphi}=\omega_{c}(z) / \omega_{0}-1,
$$

where the dot denotes the operator $\left(1 / \omega_{0}\right)(d / d t)$. Substituting Eq. (16) in Eq. (17) and normalizing the lengths and velocities to the relativistic Larmor radius $r_{L}=c / \omega_{0}$ and the light velocity $c$ respectively, we obtain

$$
\begin{aligned}
\dot{\varphi}= & \gamma^{-1}\left[\gamma_{0}-\gamma+b(\xi)\right] \\
& +g_{0} \gamma^{-1}\left(1-\gamma^{-2}-u_{z}^{2}\right)^{-1 / 2}|\sin (p \pi \xi / \delta)| \sin \varphi,
\end{aligned}
$$

where $\xi=z / r_{L}, \delta=d / r_{L}$, and $u_{z}=v_{z} / c$.

For the azimuth force component [Eq. (12)], it is more convenient to express it as an energy variation:

$$
d\left(\gamma m_{0} c^{2}\right) / d t=-e \vec{E} \cdot \vec{v} \cong-e E_{0} v_{\perp}|\sin (p \pi z / d)| \cos \varphi .
$$

Equation (19) can be rewritten as

$$
\dot{\gamma}=-g_{0}\left(1-\gamma^{-2}-u_{2}^{2}\right)^{1 / 2}|\sin (p \pi \xi / \delta)| \cos \varphi .
$$

Using expressions (3) and (17), the longitudinal force component

$$
d\left(\gamma m_{0} v_{z}\right) / d t=F_{z}
$$

can be transformed into

$$
\begin{aligned}
d\left(\gamma v_{z}\right) / d t= & -\left\{\left[v_{\perp}^{2} / 2(\dot{\varphi}+1)\right]\right. \\
& \left.-\frac{e B_{0} v_{\perp} r_{0}}{2 m_{0}} \sin (\varphi+\omega t)\right\} d b(z) / d z .
\end{aligned}
$$

From this equation, we obtain

$$
\frac{d v_{z}}{d t}=\frac{\frac{1-\gamma^{-2}-\left(v_{z} / c\right)^{2}}{2(\dot{\varphi}+1)} c^{2} \gamma^{-3} \frac{d b(z)}{d z}-\left[1-\gamma^{-2}-\left(\frac{v_{z}}{c}\right)^{2}\right]^{1 / 2} \frac{v_{z}}{c} \frac{d v_{\perp}}{d t}-\frac{\omega_{0} r_{0} c}{2}\left[1-\gamma^{-2}-\left(\frac{v_{z}}{c}\right)^{2}\right]^{1 / 2} \gamma^{-3} \sin (\omega t+\varphi) \frac{d b(z)}{d z}}{\gamma^{-2}+\left(\frac{v_{z}}{c}\right)^{2}} .
$$

Equation (15) leads to

$$
\frac{d v_{\perp}}{d t}=c\left[1-\gamma^{-2}-\left(\frac{v_{z}}{c}\right)^{2}\right]^{-1 / 2}\left[\gamma^{-3} \frac{d \gamma}{d t}-\frac{v_{z}}{c^{2}} \frac{d v_{z}}{d t}\right]
$$

Substituting Eq. (24) in Eq. (23) and normalizing the variables, we have

$$
\begin{aligned}
\dot{u}_{z}= & -\gamma^{-1}\left\{\frac{1-\gamma^{-2}-u_{z}^{2}}{2(\dot{\varphi}+1)} \frac{d b(\xi)}{d \xi}+u_{z} \dot{\gamma}\right. \\
& \left.+\frac{r_{0}^{*}}{2}\left(1-\gamma^{-2}-u_{z}^{2}\right)^{1 / 2} \sin (\varphi+\tau) \frac{d b(\xi)}{d \xi}\right\},
\end{aligned}
$$

where $r_{0}^{*}=r_{0} / r_{L}$. The function $\xi=\xi(\tau)$ is deduced from the expression 


$$
\dot{\xi}=u_{z}
$$

Equations (18), (20), (25), and (26) form a closed set of highly nonlinear differential equations which describes the time evolution of the energy, phase shift, longitudinal velocity, and $z$ position of the particle during its motion in the SARA conditions. This set can be solved numerically by using the Runge-Kutta method of the fourth order under the following initial conditions:

$$
\begin{gathered}
\varphi(0)=\pi, \\
\gamma(0)=\left[1-u_{\perp}^{2}(0)-u_{z}^{2}(0)\right]^{-1 / 2}, \\
u_{z}(0)=u_{z 0}, \\
\xi(0)=\xi_{0},
\end{gathered}
$$

where $u_{\perp}=v_{\perp} / c$.

We can turn to the cyclotron acceleration in a homogeneous magnetic field increasing slowly in time when the following substitutions will be made: $b(\xi) \rightarrow b(\tau)$, $|\sin (p \pi \xi / \delta)| \rightarrow 1$, and naturally put $u_{z}=0$. Therefore, Eqs. (18) and (20) are reduced to

$$
\begin{gathered}
\dot{\varphi}=\gamma^{-1}\left[\gamma_{0}-\gamma+b(\tau)\right]+g_{0} \gamma^{-1}\left(1-\gamma^{-2}\right)^{-1 / 2} \sin \varphi \\
\dot{\gamma}=-g_{0}\left(1-\gamma^{-2}\right)^{1 / 2} \cos \varphi
\end{gathered}
$$

These equations are similar to the equations obtained in $[5,6]$ for the GYRAC concept, except for the term corresponding to the synchrotron radiation.

When $u_{t}(0)=0$ the factor $\left(1-\gamma^{-2}-u_{z}^{2}\right)^{-1 / 2}$ causes a singularity in Eq. (18). It so happens because the definition $\omega_{c}=v_{\perp} / r$ is valid only at $r \neq 0$. To avoid the singularity at the injection point where the electric field is nil (see Fig. 1), we start with the Newton-Lorentz equation in the rectangular coordinates and use the following approximation:

$$
\begin{gathered}
\vec{B} \cong B_{0}\left[\gamma_{0}+b(z)\right] \hat{z}, \\
\gamma \cong \gamma_{0} .
\end{gathered}
$$

In this approximation, we obtain

$$
\begin{gathered}
\dot{u}_{x}=-\gamma_{0}^{-1}\left\{g_{0} \cos (\tau) \sin (p \pi \xi / \delta)+u_{y}\left[\gamma_{0}+b(\xi)\right]\right\} \\
\dot{u}_{y}=-\gamma_{0}^{-1}\left\{g_{0} \sin (\tau) \sin (p \pi \xi / \delta)-u_{x}\left[\gamma_{0}+b(\xi)\right]\right\} \\
\xi=u_{z 0} \tau .
\end{gathered}
$$

This set of differential equations can be solved with the Runge-Kutta method under the injection-point conditions: $\varphi(0)=\pi$ and $u_{x 0}=u_{y 0}=\xi=0$.

Stability of the acceleration.-Proceeding from the function $\varphi(\xi)$, which is to remain in the band $\pi / 2<$ $\varphi(\xi)<3 \pi / 2, \forall \xi$ and whose initial value is $\varphi(0)=\pi$, we can deduce the magnetic field profile $b(\xi)$ to ensure the maintenance of the acceleration regime along the electron trajectory. Hence, using Eq. (18), we get the magnetic field profile as

$$
\begin{aligned}
b(\xi)= & \left(u_{z} \frac{d \varphi}{d \xi}+1\right) \gamma-\gamma_{0}-g_{0}\left(1-\gamma^{-2}-u_{z}^{2}\right)^{-1 / 2} \\
& \times|\sin (p \pi \xi / \delta)| \sin \varphi
\end{aligned}
$$

Therefore,

$$
\begin{aligned}
\frac{d b(\xi)}{d \xi}= & \gamma\left(u_{z}^{-1} \dot{u}_{z} \frac{d \varphi}{d \xi}+u_{z} \frac{d^{2} \varphi}{d \xi^{2}}\right)+\left(u_{z} \frac{d \varphi}{d \xi}+1\right) u_{z}^{-1} \dot{\gamma}+g_{0}\left(1-\gamma^{-2}-u_{z}^{2}\right)^{-3 / 2}\left\{\left(\gamma^{-3} u_{z}^{-1} \dot{\gamma}-\dot{u}_{z}\right)|\sin (p \pi \xi / \delta)| \sin \varphi\right. \\
& \left.-\left(1-\gamma^{-2}-u_{z}^{2}\right)\left[\left(\frac{p \pi}{\delta}\right) S(\xi) \cos (p \pi \xi / \delta) \sin \varphi+|\sin (p \pi \xi / \delta)| \cos \varphi \frac{d \varphi}{d \xi}\right]\right\}
\end{aligned}
$$

Here

$$
S(\xi)=\frac{\sin (p \pi \xi / \delta)}{|\sin (p \pi \xi / \delta)|}= \begin{cases}1 & \text { if } 2 k<p(\xi / d)<2 k+1, k=0,1,2, \ldots \\ 0 & \text { if } p(\xi / d)=k \\ -1 & \text { if } 2 k+1<p(\xi / d)<2 k+2, k=0,1,2, \ldots\end{cases}
$$

For the particular case of $r_{0}^{*}=0$, substituting Eq. (39) in Eq. (25), we have

$$
\begin{aligned}
\dot{u}_{z}= & \left\{\frac { ( 1 - \gamma ^ { - 2 } - u _ { z } ^ { 2 } ) } { 2 ( u _ { z } \frac { d \varphi } { d \zeta } + 1 ) } \left\langleu_{z} \frac{d^{2} \varphi}{d \xi^{2}}+\gamma^{-1}\left[\left(u_{z} \frac{d \varphi}{d \xi}+1\right) u_{z}^{-1} \dot{\gamma}+g_{0} \gamma^{-3}\left(1-\gamma^{-2}-u_{z}^{2}\right)^{-3 / 2} u_{z}^{-1}\left|\sin \left(\frac{p \pi \xi}{\delta}\right)\right| \sin \varphi \dot{\gamma}\right.\right.\right. \\
& \left.\left.\left.+g_{0}\left(1-\gamma^{-2}-u_{z}^{2}\right)^{-1 / 2}\left((p \pi / \delta) S(\xi) \cos \left(\frac{p \pi \xi}{\delta}\right) \sin \varphi+\left|\sin \left(\frac{p \pi \xi}{\delta}\right)\right| \cos \varphi \frac{d \varphi}{d \xi}\right)\right]\right\rangle+u_{z} \gamma^{-1} \dot{\gamma}\right\} \\
& \times\left[\frac{\left(1-\gamma^{-2}-u_{z}^{2}\right)}{2\left(u_{z} \frac{d \varphi}{d \zeta}+1\right)}\left(-u_{z}^{-1} \frac{d \varphi}{d \zeta}+g_{0} \gamma^{-1}\left(1-\gamma^{-2}-u_{z}^{2}\right)^{-3 / 2}\left|\sin \left(\frac{p \pi \xi}{\delta}\right)\right| \sin \varphi\right)-1\right]^{-1} .
\end{aligned}
$$


Solving the differential equations (20), (26), and (41) at the initial conditions (28)-(30), we can, first, obtain the functions $\gamma, u_{z}$, and $\xi$, and then the magnetic field profile can be calculated using Eq. (38). The singularity in Eq. (41) at $b(\xi) \cong 0$ is treated in the same way as the Eq. (18) singularity.

A continuous sustenance of the exact resonance is possible only in the particular case of $p=1$, because, if $p \neq$ 1 , the phase $\varphi$ jumps an angle $\pi$ in each node of the standing electromagnetic wave. For the exact resonance $\varphi=\pi$, Eq. (38) leads to

$$
\gamma=\gamma_{0}+b
$$

Substituting Eq. (42) in Eqs. (20) and (41), for the case of $\varphi=\pi$, we obtain

$$
\begin{aligned}
& \dot{b}=g_{0}\left[1-\left(\gamma_{0}+b\right)^{-2}-u_{z}{ }^{2}\right]^{1 / 2} \sin [\pi \xi(\tau) / \delta], \\
& \dot{u}_{z}=(1 / 2)\left(\gamma_{0}+b\right)^{-1}\left\{\left[\left(\gamma_{0}+b\right)^{-2}-1\right] u_{z}^{-1}-u_{z}\right\} \dot{b},
\end{aligned}
$$

where $\dot{b}=u_{z}(d b / d \xi)$, and $u_{z}$ are determined by expression (26). Equations (43) and (44) can be solved numerically with the conditions at the injection point $z=0$ :

$$
\begin{gathered}
b(0)=0, \\
u_{z}(0)=u_{z 0}, \\
\xi(0)=0 .
\end{gathered}
$$

The simulations are stopped when the electron comes in contact with the cavity wall or reaches its return point on a stop plane where $u_{z}=0$ ( 7 in Fig. 1). The latter condition generates a singularity in Eq. (44), and therefore the minimum $z$ velocity at the return point is taken as $u_{z \min }=$ $10^{-4}$. The corresponding total electron energy can be obtained directly from Eq. (42).

For the case of $p=2$, the phase shift in the microwave field node, which divides the cavity into two parts, is changed by $\pi$. The fulfillment of the expression

$$
\varphi(\xi)= \begin{cases}2 \pi-\frac{\pi}{1+\exp [10(\xi-\delta / 2) \Delta \xi]} & \text { if } 0 \leq \xi<\delta / 2 \\ \pi-\frac{\pi}{1+\exp [10(\xi-\delta / 2) / \Delta \xi]} & \text { if } \delta / 2<\xi \leq \delta\end{cases}
$$

guarantees the maintenance of the acceleration regime. Here $\delta$ and $\Delta \xi$ are the normalized lengths of the cavity and of the zone where the phase shift varies, respectively. We can note that $\varphi(0)=\pi$ and $\left.\varphi\right|_{(\delta / 2)^{-}}-\left.\varphi\right|_{(\delta / 2)^{+}}=\pi$.

\section{RESULTS AND DISCUSSIONS}

Now we proceed from the case $B_{z}(z)=B_{z}(0, z)$ which was studied in our previous work [13]. Using the values obtained in [13], the function $b\left(z_{i}\right)=B\left(0, z_{i}\right) / B_{0}-1$ was determined on the $z$ axis in 81 points where the derivative $d b\left(z_{i}\right) / d z$ was found numerically. The values $b(z)$ and

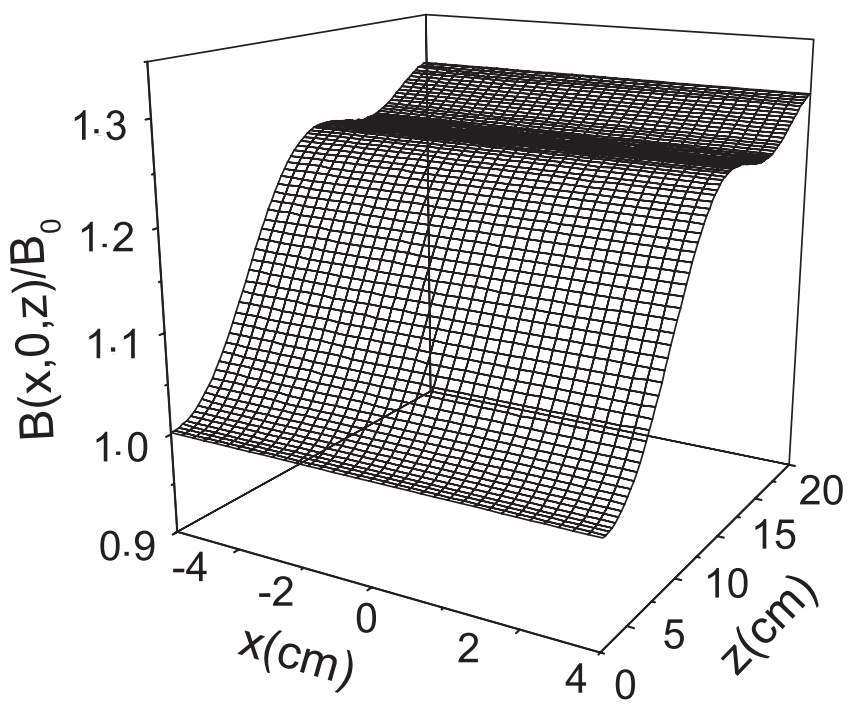

FIG. 4. The magnetic field profile in the $y=0$ plane.

$d b(z) / d z$ in the particle position points were calculated by using a linear interpolation method. The magnetic field shown in Fig. 4 is determined in the approximation of Eq. (3). In our case, the radial component $B_{r}(r, z)$ expressed through the first term in the right-hand side of Eq. (2), is insignificant in comparison with the longitudinal component. Accordingly, the radial inhomogeneity of the magnetic field is found equal to $(1 / 8) B_{0} r^{2}[d b(z) / d z]^{2} /\left[\gamma_{0}+b(z)\right]$. In spite of this, the transversal component can hardly be ignored because the diamagnetic force depends on this component. It should be mentioned in this connection that the longitudinal velocity $u_{z}$ of the electron injected at $r_{0}=1.5 \mathrm{~cm}$ oscillates around the values calculated for the electron which begins to move along the cavity axis but this oscillations are found insignificant, $\Delta u_{z} / u_{z}<4 \times 10^{-2}$. The energy difference is found still smaller. This is why the graphs demonstrating the mentioned differences are not shown.

Figure 5 shows the energy space evolution derived for the present model and for the magnetic field data given in [13]. Both simulations were fulfilled for the electrons with an initial energy of $10 \mathrm{keV}$ injected into the cavity where a $\mathrm{TE}_{112}$ microwaves of $2.45 \mathrm{GHz}$ frequency and an amplitude of $6 \mathrm{kV} / \mathrm{cm}$ was excited. The phase-shift dependence on the longitudinal coordinate for the cases of Fig. 5 is presented in Fig. 6.

The difference between the energies (see Fig. 5) which is observed in the space $z \geq 12.5 \mathrm{~cm}$ where the Larmor radius is relatively large $(0.7-1.6 \mathrm{~cm})$, can be attributed to the difference in the transversal magnetic field inhomogeneities. In case [13], the diamagnetic force is high enough to prevent the electron from crossing the $z=$ $19 \mathrm{~cm}$ transverse plane (Fig. 5, the circles) whereas in the case of the approximated magnetic field the acceleration in the transversal plane is not so effective that it 


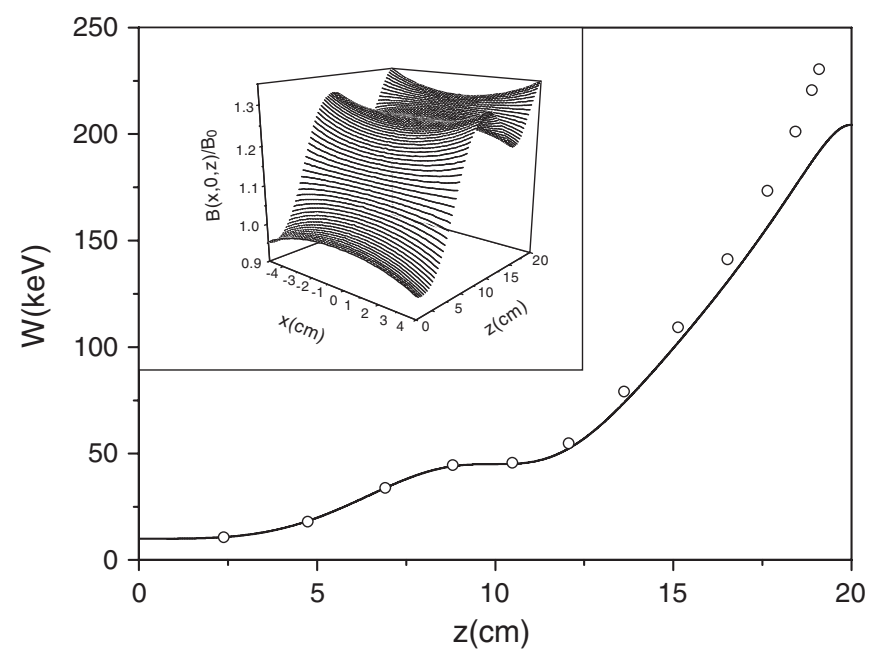

FIG. 5. Spatial evolutions of the electron energy: the solid line is the SARA model, the circles represent the data obtained in Ref. [13].

permits the electron to achieve the lateral cavity wall (Fig. 5, the solid line).

Figure 7 demonstrates the electron energy evolution calculated on the basis of the SARA model (solid, dashed, and doted lines) and the exact equation data (circles, triangles, and rectangles) at the initial energies of 10, 20, and $30 \mathrm{keV}$. The other experimental parameters are as follows: the cylindrical cavity of $2.0 \mathrm{~m}$ long with a radius of $1.33 \mathrm{~m}$ was excited at $\mathrm{TE}_{111}$ mode by $100 \mathrm{MHz}$ microwaves with the amplitude of $6 \mathrm{kV} / \mathrm{cm}$. The space variation of the magnetic field is determined by a linear function $b(z)=\alpha z$, where $\alpha=\gamma_{0}\left(R_{m}-1\right) / d$ and $R_{m}=1.3$. One can see that the SARA model data agrees well with the exact equation simulation results. The electron with the initial energy of $30 \mathrm{keV}$ penetrates deeper into the cavity

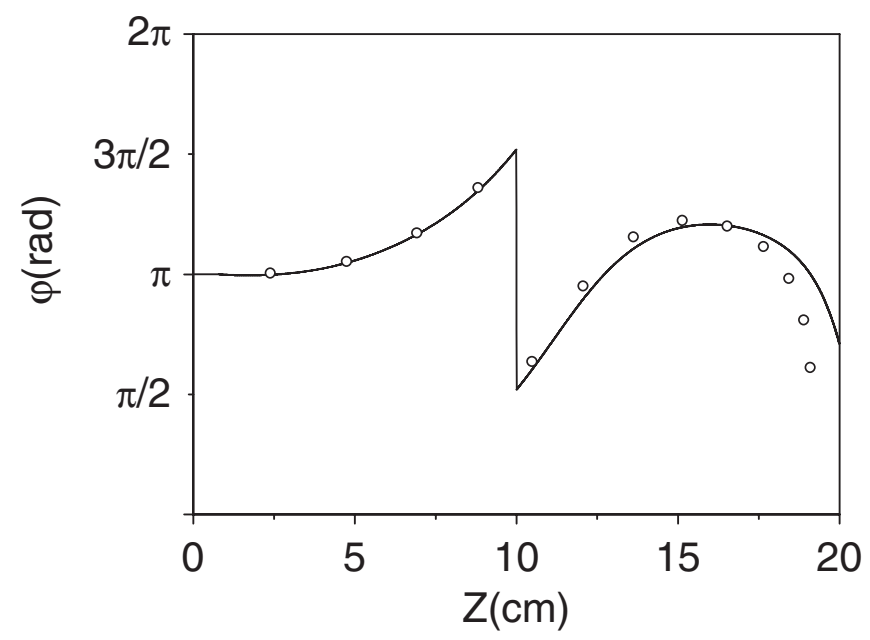

FIG. 6. Dependence of the phase shift on the longitudinal coordinate: the solid line is the SARA model; the circles represent data obtained in Ref. [13].

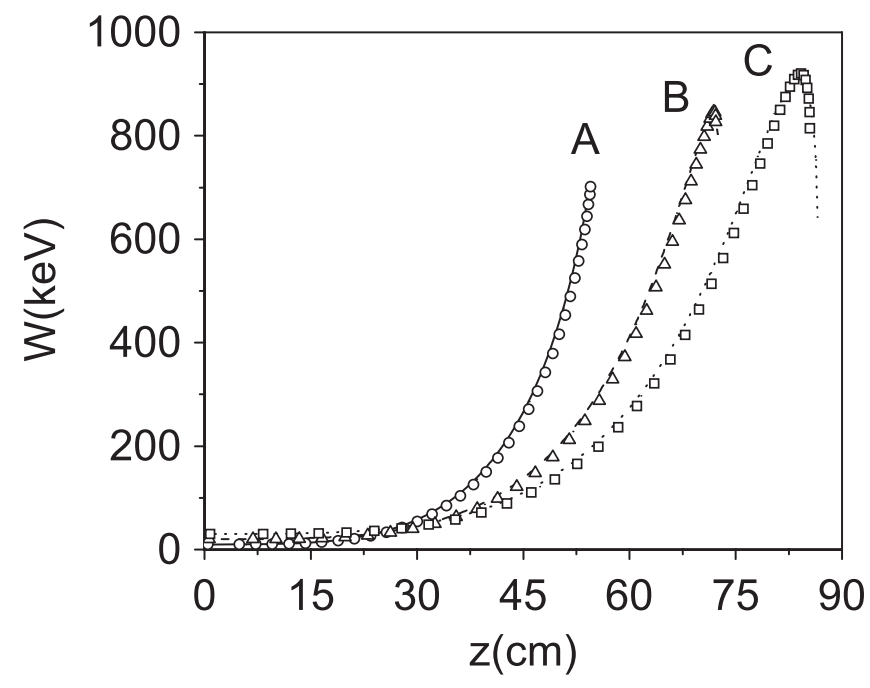

FIG. 7. Energy evolution in a linear magnetic field at different initial injection energies: (A) $W_{i}=10 \mathrm{keV}$, (B) $W_{i}=20 \mathrm{keV}$, (C) $W_{i}=30 \mathrm{keV}$.

than the electrons with lower initial energies and speeds up to $0.94 \mathrm{MeV}$.

Figure 8 shows the calculations fulfilled in accordance with the SARA model for a magnetic profile $b(z)=\alpha z^{2}$, $\alpha=\gamma_{0}\left(R_{m}-1\right) / d^{2}$, and $R_{m}=1.2$. The cavity parameters were taken identical with those used for the linear case but microwave field was chosen equal to $10 \mathrm{kV} / \mathrm{cm}$. In this case, the electron with the initial energy of $30 \mathrm{keV}$ is accelerated up to $1.2 \mathrm{MeV}$. The electron is seen to acquire more energy in a magnetic field of a parabolic type than in the linear case.

Figure 9 shows the evolution of the phase shift, determined by expression (48), along the electron path for three phase zone lengths $\Delta z=12 \mathrm{~cm}, 9 \mathrm{~cm}$, and $6 \mathrm{~cm}$. At the

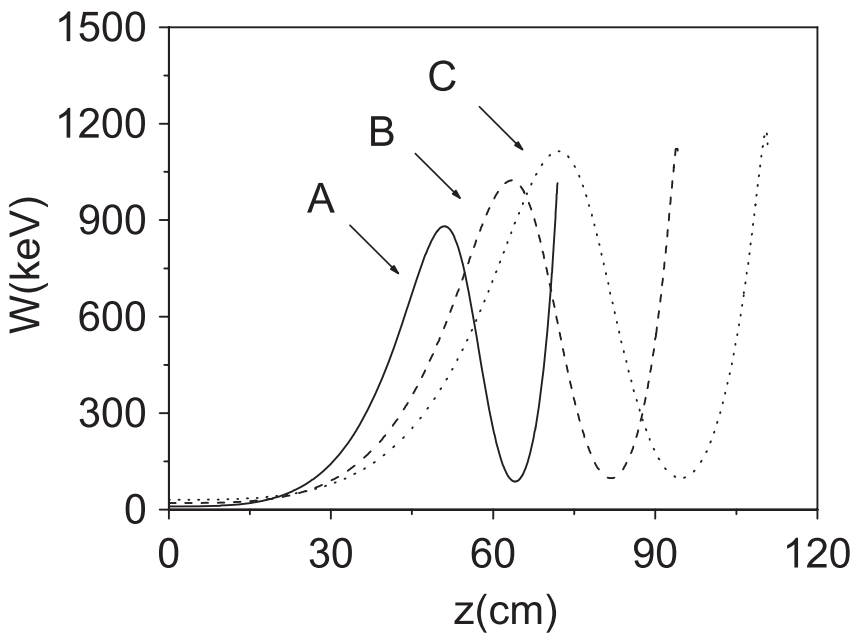

FIG. 8. Electron energy evolution at different initial injection energies for a parabolic magnetic field case: (A) $W_{i}=10 \mathrm{keV}$, (B) $W_{i}=20 \mathrm{keV}$, (C) $W_{i}=30 \mathrm{keV}$. 


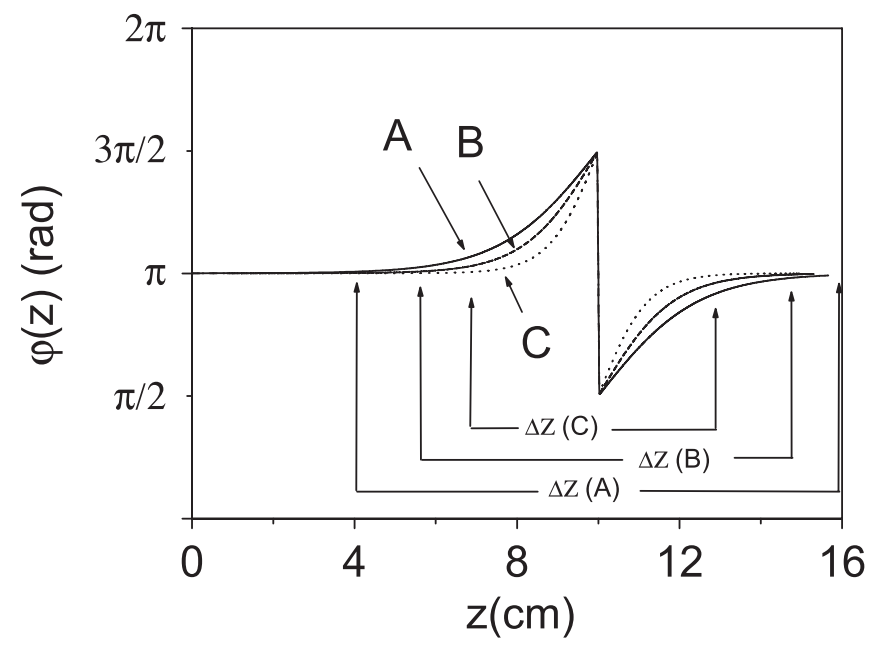

FIG. 9. Dependence of the phase shift on the longitudinal coordinate for different lengths $\Delta z$ of the zone where the phase shift varies: (A) $\Delta z=12 \mathrm{~cm}$, (B) $\Delta z=9 \mathrm{~cm}$, (C) $\Delta z=6 \mathrm{~cm}$.

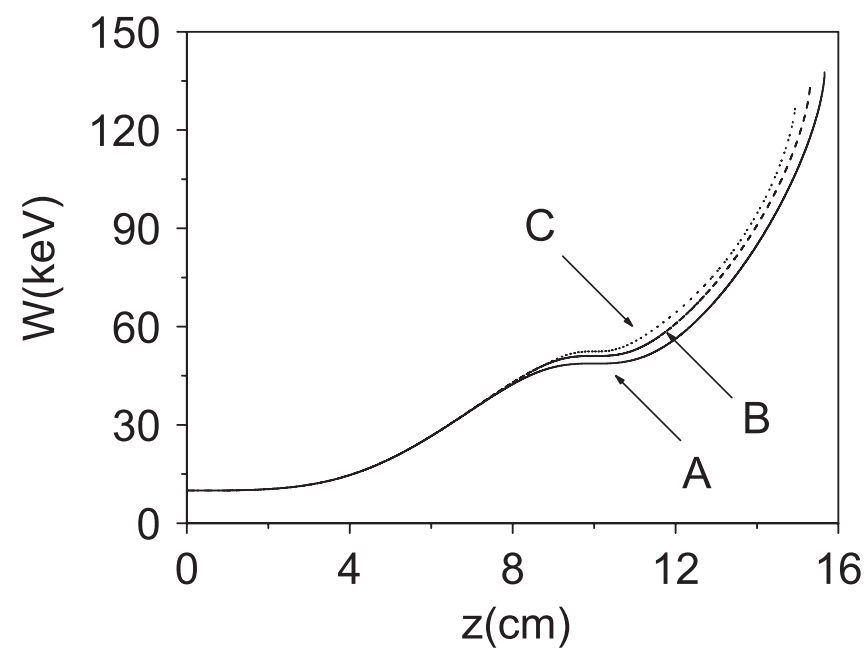

FIG. 10. Electron energy space evolution for different lengths of the zone where the phase shift is varied: (A) $\Delta z=12 \mathrm{~cm}$, (B) $\Delta z=9 \mathrm{~cm}$, (C) $\Delta z=6 \mathrm{~cm}$.

injection point, the electron is in the exact cyclotron resonance which is followed by the phase-shift deviation from $\pi$. However, this deviation remains in the acceleration band, which guarantees the acceleration stability.

The smaller is $\Delta z$, the larger is the zone where the electron is found in the near-exact cyclotron resonance conditions. Figure 10 shows the electron energy change for the $\Delta z$ values, presented in Fig. 9. The energy increases monotonically with the exception of the region close to $z=10 \mathrm{~cm}$ where the microwave field is nil. The energy rise comes to a stop when the diamagnetic force prevents the electron from penetrating into a higher magnetic field region (see Fig. 10). It is worth mentioning in this connection that the optimization of $\varphi(z)$ for the maximum energy achievement is not discussed in the present work.

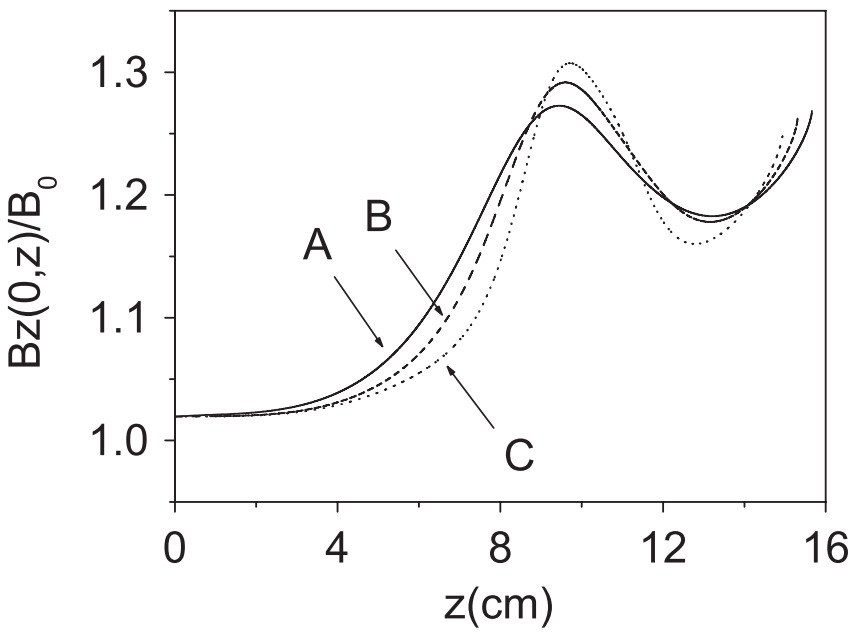

FIG. 11. Magnetic field profile for different lengths of the zone where the phase shift varies: (A) $\Delta z=12 \mathrm{~cm}$, (B) $\Delta z=9 \mathrm{~cm}$, (C) $\Delta z=6 \mathrm{~cm}$.

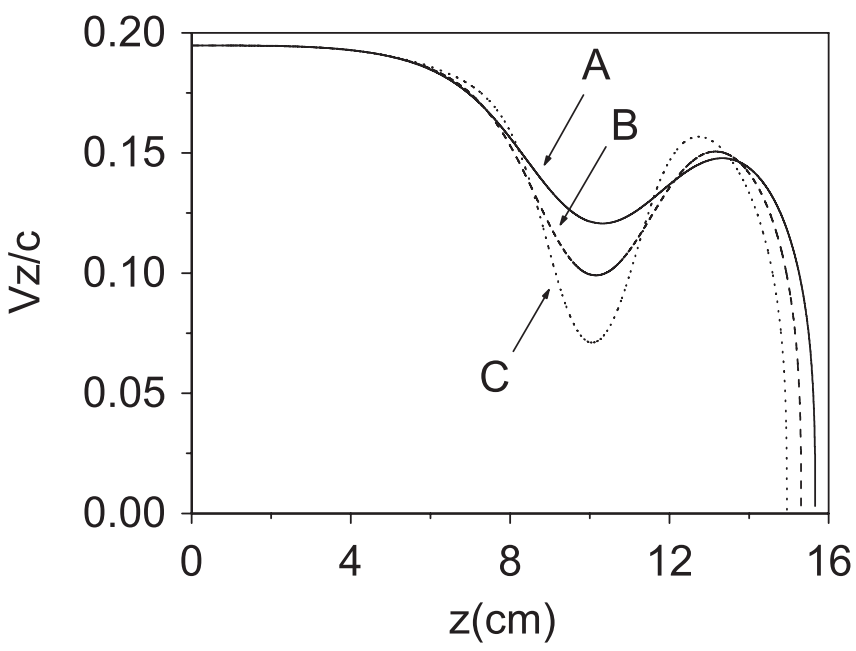

FIG. 12. Longitudinal velocity evolution for different lengths of the zone where the phase shift varies: (A) $\Delta z=12 \mathrm{~cm}$, (B) $\Delta z=9 \mathrm{~cm}$, (C) $\Delta z=6 \mathrm{~cm}$.

The magnetic field profiles for different $\Delta z$ presented in Fig. 11 were calculated using expression (38). The results shown in Fig. 11 offer the magnetic field profiles which provide quite an effective acceleration along the electron trajectory. Figure 12 shows the longitudinal velocity component evolution as the function of the electron $z$ coordinate for each case presented in Figs. 10 and 11. At the end of the electron motion, the longitudinal velocity diminishes or falls to zero due to the diamagnetic force action.

\section{CONCLUSIONS}

The possibility of the cyclotron autoresonance acceleration in space inhomogeneous magnetic fields is analyzed. The magnetic field configuration which keeps the condi- 
tions for the phase shift up in the acceleration band is found.

The total SARA operation parameters are of steady-state mode and make the SARA concept more attractive for practical realizations if compared with the existing selfsustained acceleration methods. The problems of how the synchrotron radiation force influences the electron energy balance and of acceleration stability with respect to the initial conditions will be analyzed in our future work.

\section{ACKNOWLEDGMENTS}

This work was supported by the Universidad Industrial de Santander, Colombia. One of the authors (E. A. O.) is grateful to Colciencias (Colombia) for financial support.

[1] A. A. Kolomenskii and A. N. Levedev, Dokl. Akad. Nauk USSR 145, 1251 (1962) [Sov. Phys. Dokl. 7, 492 (1962)].
[2] V. Ya. Davydovskii, Zh. Eksp. Teor. Fiz. 43, 886 (1962) [Sov. Phys. JETP 16, 629 (1963)].

[3] H. R. Jory and A. W. Trivelpiece, J. Appl. Phys. 39, 3053 (1968).

[4] C. Roberts and S. Buchsbaum, Phys. Rev. 135, A381 (1964).

[5] K. S. Golovanivsky, IEEE Trans. Plasma Sci. 11, 28 (1983).

[6] O. Gal, IEEE Trans. Plasma Sci. 17, 622 (1989).

[7] C. Chen, Phys. Fluids B 3, 2933 (1991).

[8] A. P. Ishkov, Izv. Vusov Fisica 2, 176 (1970) (in Russian).

[9] R. Shpitalnik, C. Cohen, F. Dothan, and L. Friendland, J. Appl. Phys. 70, 1101 (1991).

[10] L. Friendland, Phys. Plasmas 1, 421 (1994).

[11] V. D. Dougar-Jabon, A. M. Umnov, and D. Suescun Díaz, Rev. Sci. Instrum. 73, 629 (2002).

[12] K. F. Sergeichev, D. M. Karfidov, and N. A. Lukina, Plasma Phys. Rep. 33, 455 (2007).

[13] V.D. Dougar-Jabon, E. A. Orozco, and A. M. Umnov, Phys. Rev. ST Accel. Beams 11, 041302 (2008). 